PAPER

\title{
Comparison of obstacle sense ability between the blind and the sighted: A basic psychophysical study for designs of acoustic assistive devices
}

\author{
Takahiro Miura ${ }^{1,2, *}$, Teruo Muraoka ${ }^{2, \dagger}$ and Tohru Ifukube ${ }^{2, \dagger}$ \\ ${ }^{1}$ Graduate School of Information Science and Technology, the University of Tokyo, \\ 7-3-1, Hongo, Bunkyo-ku, Tokyo, 113-8654 Japan \\ ${ }^{2}$ Research Center for Advanced Science and Technology, the University of Tokyo, \\ 4-6-1, Komaba, Meguro-ku, Tokyo, 153-8904 Japan
}

(Received 19 May 2009, Accepted for publication 1 July 2009)

\begin{abstract}
The ability by which the blind can recognize objects around them solely by hearing is called "obstacle sense." By analyzing and modeling the mechanism of this sense, the resultant model could be utilized in new concepts for blind mobility aids as well as training methods. In this paper, we first conducted a comparative experiment regarding coloration perception between the blind and the sighted. In the experiment, subjects are asked to answer whether two successive sounds with a different dip-to-dip interval are perceived to be same by means of two alternative forced choices. The results show no significant difference in discrimination between the two groups; the blind and the sighted. Next, "impressions" elicited by the sounds with various dip-to-dip intervals are analyzed on the two groups using the Semantic Differential Method (SDM). The results indicate that the sighted tend to focus mainly on the quantitatively represented changes such as pitch and loudness of the sounds, while the blind are inclined to focus not only on the quantitative sound change, but also on qualitative impressions in the sound changes. Since it is assumed that the qualitative impressions are related to distances of the obstacles from the blind, third, we carry out a comparative experiment regarding the obstacle-distance perception. The result indicates that the blind can more exactly answer the obstacle-distance than the sighted. From the results of the three experiments and past studies, we discuss whether the obstacle sense is formed in the peripheral process or in the central process of the auditory nervous system, and then we propose new concepts for blind mobility aids as well as the obstacle sense training method.
\end{abstract}

Keywords: Obstacle sense, Blind, Coloration, Ripple noise, DL, Semantic Differential Method

PACS number: 43.66.Fe, 43.66.Lj [doi:10.1250/ast.31.137]

\section{INTRODUCTION}

A large number of special education methods [1] and blind mobility aids [2-6] have been proposed to help the blind in walking and in carrying out other routine activities. Walking training is divided into two phases: "orientation" that is involved in awareness of one's position in the environment; and "movement" that is involved in movement toward one's direction [1]. Although the blind cannot use their visual sense, it is known that most of them can localize themselves in the environment by using "obstacle sense," which is the ability that enables them to perceive objects solely by hearing [1,7-10]. It has been reported

\footnotetext{
*e-mail: miura@human.rcast.u-tokyo.ac.jp

†e-mail: muraoka@human.rcast.u-tokyo.ac.jp

‡e-mail: ifukube@human.rcast.u-tokyo.ac.jp
}

that some auditory-enhanced blind people can detect an object's existence, and also can estimate its distance, size and shape [11-14]. We have analyzed the auditory mechanism of the obstacle sense based on psychophysical experiments and acoustic signal processing methods in order to propose a new blind mobility aids. Our concept of the mobility aids is to positively utilize the obstacle sense, different from conventional mobility aids that rather prevent the blind from using their obstacle sense ability [2].

In this paper, first, we present an overview of previous studies on obstacle sense. This ability was introduced, by Diderot in 1749, as "amazing ability" of a blind to judge accurate distance of the object from him as well as to perceive the presence of object without visual information [15]. Many cases and theories related to this ability had been reported since the publication of his account. Hayes summarized these theories into three groups such as 
sensory, perceptual, and occult theories [16]. The sensory theories postulate a enhanced response of some organs of sense such as the sense of a face for pressure or temperature, of tympanic membranes in the ears for pressure, or of audition in the increased discrimination and analysis of the sound. The perceptual theories include the interpretation of sensory cues derived from the action of the air- or soundwaves on the skin or aural mechanisms. The occult theories explain this phenomenon is related to the action of vestigial organs in the skin and of the subconscious caused by magnetism, electricity, vibration of the ether or some their hypothetical substance. These hypotheses were organized on the basis of introspection by the blind as two theories; sense of the skin and of the auditory by Supa et al. [7].

Supa et al. also performed systematic studies of obstacle sense among the blind. They reported that the factor behind obstacle sense was not due to skin sensation, but resulted from auditory stimuli [7], in which the stimuli of the external ear were not important [8]. Cotzin et al. reached the assumption that auditory stimuli-induced obstacle sense was caused by sounds reflected from the obstacle [9]. Seki et al. particularly focused on "acoustic coloration," which is caused by the interference between the direct sound and the reflected sound from the obstacle. They reported that the change in pitch resulting from the acoustic coloration is an essential factor in perceiving the distance from the subject to the object [10]. This interference makes sound nearly equal to comb-filtered direct sound. In a comb-filter, the dip-to-dip distance in frequency region is defined as the dip-frequency interval, which changes depending on the distance between the subject and the obstacle [18].

It also has been reported that comb-filtered sound produces pitches (sometimes referred to as repetition pitches) similar to residue pitches or missing fundamental pitches [19-25]. In particular, Yost et al. precisely investigated the characteristics of comb-filtered noise, and they referred the noise as ripple noise. It is reported that the ripple noise has a matched pitch equal to the reciprocal of the delay [20]. They reported that a transformation of the ripple noise spectrum based on a peripheral weighting function produces a consistent spectrum pattern as repetition pitch and pitch strength [19]. Ando showed that the perception of coloration in continuous sound fields results from the autocorrelation function of the signal in the short delay range of a weak sound [26]. Kates proposed a central auditory spectrum processing model for coloration perception in ripple noise [27]. Krumbholz investigated the salience pitch associated with a ripple noise when interaural time and level differences occur [28]. Although these results and assumptions only explain the common perception of a complex sound pitch, they cannot explain how this perception contributes to obstacle sense of the blind.
On the other hand, our approach is to investigate how the pitch perception of the ripple noise contributes to obstacle sense for both the blind and the sighted in order to clarify the auditory mechanism differences between the two. Furthermore, our goal is to construct a training method of the obstacle sense for the blind and also design a blind mobility aid that positively use the obstacle sense ability. If the blind are superior to the sighted in terms of pitch discrimination ability of ripple noise, they have an advantage to acquire the obstacle sense on a level of timbre discrimination that would be realized in the peripheral auditory system. In contrast, if the sighted have the same discrimination ability as the blind, it would be possible for them to acquire the obstacle sense only using auditory stimuli such as intensity, reflected sounds, coloration and other kinds of sound characteristics [9,10,14,17].

In this paper, we first conducted a comparative experiment regarding coloration perception between the blind and the sighted. In the experiment, subjects are asked to answer whether two successive sounds with a different dipto-dip interval are perceived to be same by means of two alternative forced choices. The results show no significant difference in discrimination between the blind and the sighted. Next, "impressions" elicited by the sounds with various dip-to-dip intervals are analyzed on the two groups using the Semantic Differential Method (SDM). The results indicate that the sighted tend to focus mainly on the quantitatively represented changes such as pitch and loudness of the sounds, while the blind are inclined to focus not only on the quantitative sound change, but also on qualitative impressions in the sound changes. Since it is assumed that the qualitative impressions are related to distances of the obstacles from the blind, third, we carry out a comparative experiment regarding the obstacledistance perception. The result indicates that the blind can more exactly answer the obstacle-distance than the sighted. From the results of the three experiments and past studies, we discuss whether the obstacle sense is formed in the peripheral process or in the central process of the auditory nervous system, and then we propose new concepts for blind mobility aids as well as the obstacle sense training method.

\section{COLORATION}

A timbre by coloration is caused by the interference between two surrounding sounds around a subject [29]. One is a surrounding sound directly transmitted to the subject's ears, the other is a reflected sound from obstacles existing in the same surroundings. A time delay between the two sound waves at the subject's ears forms a comblike spectral pattern with dips. The frequency difference between the dips is a reciprocal of the time delay. Therefore, the coloration is an important information cue 


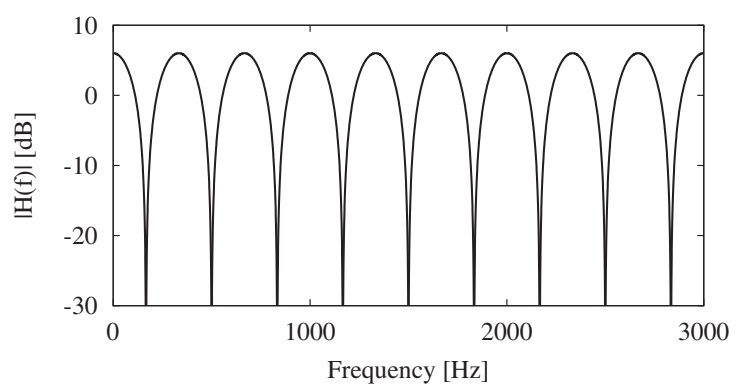

Fig. 1 An example of spectrum patterns with periodical dips that cause acoustic coloration $(A=1, T=3 \mathrm{~ms})$.

for identifying the distance from the obstacle to the subject [18]. A spectral amplitude of a synthesized sound wave consisting of direct and reflected sound wave $|H(f)|$ is expressed by:

$$
|H(f)|=\sqrt{1+A^{2}+2 A \cos 2 \pi f T}
$$

where $f, A$, and $T$ stand for frequency, reflectivity coefficient of obstacle, and arrival-time interval between the direct and reflected sound, respectively. Figure 1 is an example of visual representation for (1).

Here, the dip-to-dip interval in the spectrum is defined as the dip-frequency interval $\Delta f_{d}$, the relation between $\Delta f_{d}$ and the distance from obstacle $d$ is:

$$
d=\frac{c}{2 \Delta f_{d}}
$$

where $c$ stands for sound velocity. The relationship between the obstacle distance from an arbitrary position and dip-frequency intervals is shown in Fig. 2. In the experiments described below, we especially focus on the discrimination threshold of $\Delta f_{d}$ and acoustic impressions regarding the change in $\Delta f_{d}$.

(1) is the same equation as the frequency response of the comb-filter. Thus, sound waves caused by the coloration can be generated by applying a comb-filter to a signal. In the real world, the blind can recognize the coloration by listening to the surrounding noise. Thus, we use the combfiltered noise, which is known as ripple noise [19,20,24]. It has been reported that the ripple noise has a matched pitch equal to the inverse of the time delay, and produces pitches (sometimes referred to repetition pitches) similar to residue pitches or missing fundamental pitches [20,25].

\section{DISCRIMINATION EXPERIMENT FOR THE CHANGE OF DIP-FREQUENCY INTERVAL}

\subsection{Subjects}

Six congenital visually impaired people aged 23-34 years old participated in the following experiments. Two of them are congenitally blind, the other four are acquired blind. Acquired blind subjects congenitally had visual

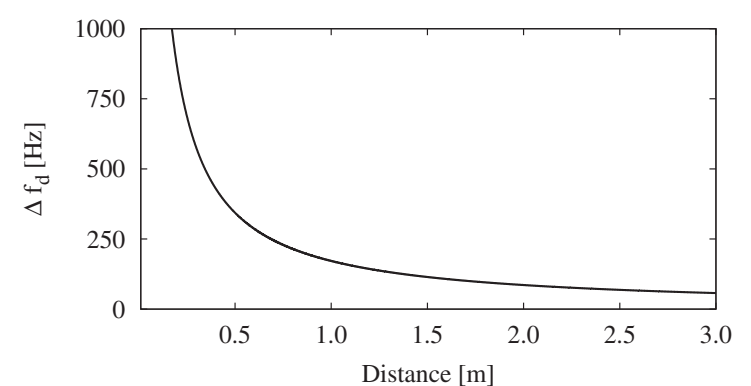

Fig. 2 Dip-frequency interval change in relation to the distance between the obstacle and the observation point.

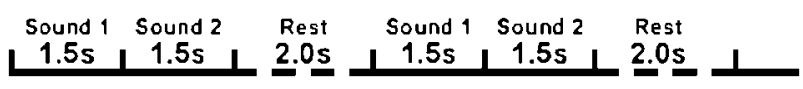

Fig. 3 Experimental protocol for discrimination of spectrum with different $\Delta f_{d}$. Sound $1\left(f_{d}\right)$ varies in the range of $50,60,100,200,300,400,500,600$ and $1,000 \mathrm{~Hz}$, while Sound 2 changes in the range of $0, \pm 0.1, \pm 0.2, \pm 1, \pm 2, \pm 5, \pm 10, \pm 20, \pm 50 \mathrm{~Hz}$ around Sound 1 .

disorders such as amblyopia and had been depended on as well as visual information before getting totally blind in midstream. Of the six, four are totally blind, while two can only recognize the existence of light stimuli. Four are males and two are females. As the sighted control group, four males and two females aged 23-42 years old participated in the experiment. All of the subjects have normal hearing; within normal limits $(-20 \mathrm{~dB} \mathrm{HL})$ as defined by pure-tone audiometry. In addition, experimenter explains the contents of the experiment to subjects and all subjects agree to participation.

\subsection{Methods}

Before the experiment starts, we asked the subjects to put on headphones and adjust the sound to a comfortable level. Then, two successive sounds are presented at the time sequence as shown in Fig. 3. Sound 1 of $\Delta f_{d}$ varies in the frequency range of 50,60, 100, 200,300,400, 500, 600 and $1,000 \mathrm{~Hz}$, while Sound 2 varies $0, \pm 0.1, \pm 0.2, \pm 1, \pm 2$, $\pm 5, \pm 10, \pm 20, \pm 50 \mathrm{~Hz}$ around Sound 1 . These sounds are comb-filtered white noises (frequency range: $20 \mathrm{~Hz} \sim$ $20 \mathrm{kHz}$ ). Sounds like white noise are ordinarily existing in the real acoustic environment and these sounds result in lower localization errors than sinusoidal sounds [30]. The experiment is performed based on a two-alternative forced choice task, and the subjects are asked to answer whether the two sounds are same or not by inputting 0 or 1 via a keyboard. In addition, the subjects are asked to inform their mistyping so that typing errors are corrected by the experimenter and are asked to close their eyes in experiment for concentrating on the series of stimuli. 


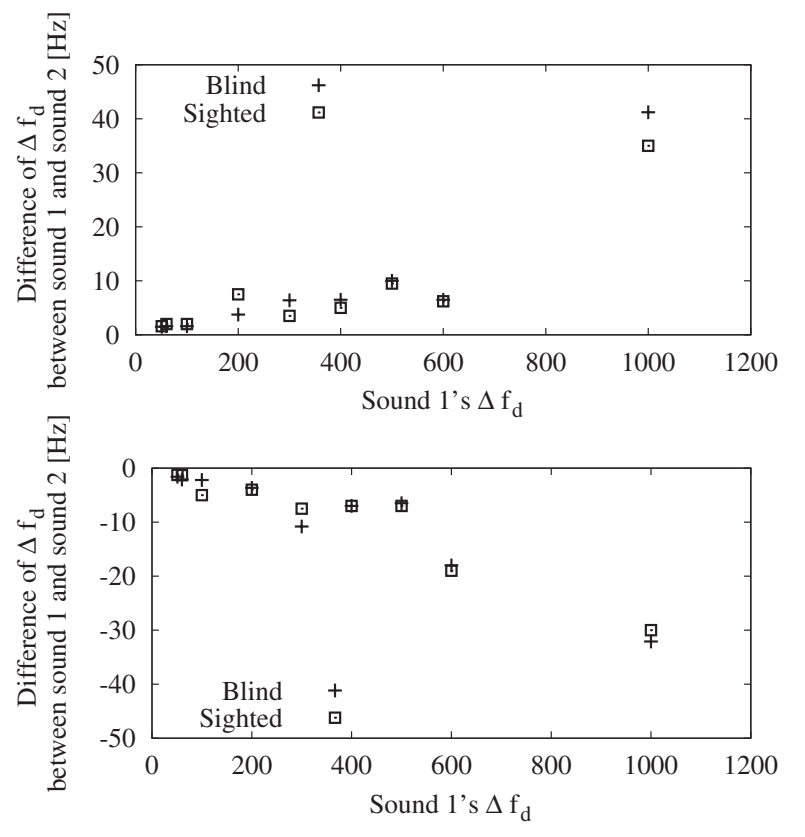

Fig. 4 Discrimination results of dip-frequency interval $\left(\Delta f_{d}\right)$ changes as a function of $\Delta f_{d}$. The top graph shows the data in the case of an increase in dipfrequency interval; the bottom graph shows those in the case of a decrease. The results show very few differences between the blind and the sighted.

The experiment is composed of 20 sets for training and 200 sets for actual tests. In actual tests, set of $\Delta f_{d}$ in sound 1 consists of above-mentioned nine and randomly-selected one frequency range (total: 10 sets). Series of randomlyselected one stimuli are firstly presented but count out from the result. Whereas, sound 2 varied 0 (4 times), $\pm 0.1, \ldots, \pm 50 \mathrm{~Hz}$ around sound 1 (total: 20 sets).

The difference limen is defined as over $75 \%$ in the accuracy ratio for each set of Sound 1 and Sound 2, and determined by linear approximation.

\subsection{Results}

Figure 4 shows the result of the $\Delta f_{d}$ discrimination test represented by DL (difference limen) between the blind and the sighted. The top graph shows the result in the case of a $\Delta f_{d}$ increase around the frequency of Sound 1; the bottom graph shows the result in the case of a $\Delta f_{d}$ decrease. These graphs show there is little difference in $\Delta f_{d}$ change discrimination between the blind and the sighted. In addition, the discrimination difference between the increase and the decrease of the dip-frequency interval is properly observed over $600 \mathrm{~Hz}$ in the dip-frequency interval of Sound 1.

Figure 5 shows the perceivable distance ratio between the corresponding distance change to difference limen for the dip-frequency interval and the distance from the obstacle by blind subject group; the result of sighted group is almost same. The perceivable corresponding distance

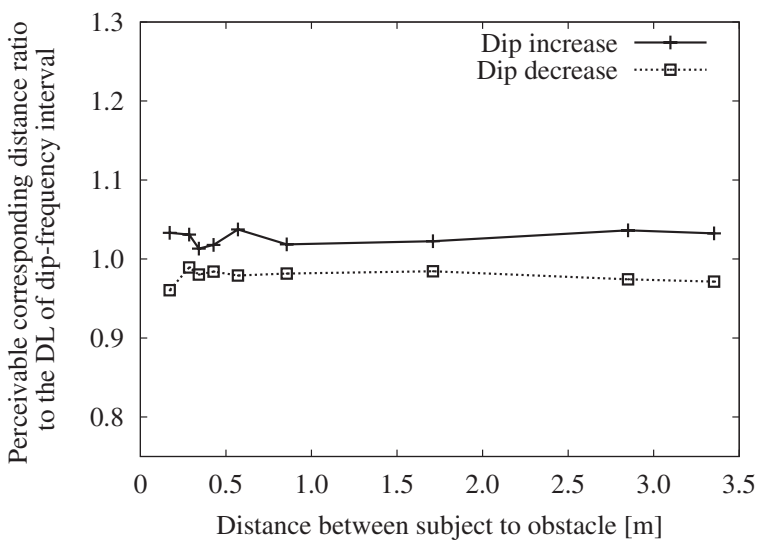

Fig. 5 Differentiable distance ratio between the corresponding distance change to difference limen for dipfrequency interval of the blind and the distance from the obstacle. The continuous line plots the case of a dip-frequency interval increase; the dotted line plots the case of a decrease.

ratios to the DL of increasing and decreasing dip-frequency intervals are $0.960 \sim 0.984$ (Mean: 0.978, S.D.: 0.00858) and $1.013 \sim 1.037$ (Mean: 1.027, S.D.: 0.00896), respectively in the blind, and $0.966 \sim 0.990$ (Mean:0.977, S.D.: 0.0104 ) and $1.014 \sim 1.053$ (Mean:1.027, S.D.: 0.0113) in the sighted.

\subsection{Discussion}

The results shown in Fig. 4 suggest there is little difference between the blind and the sighted in difference limen for dip-frequency difference interval. Bross and Borenstein reported almost the same hearing-threshold levels for the blind as for the sighted [31]. Starlinger and Niemeyer found there is no significant difference in perception of loudness and frequency changes between the sighted and the blind [32]. Benedett and Loeb also found no significant difference in basic auditory sensitivity between the two groups [34]. Thus, it is ascertained that there is no significant difference in peripheral auditory function regarding the dip frequency discrimination between the blind and the sighted. In addition, the superiority of the blind in obstacle sense is mainly caused not by sensory compensation, but by perceptual compensation, which is influenced by continuous auditory training [12,33-35]. Therefore, it is likely that auditory obstacle sense likewise results from sensitization not of the peripheral nervous system but of the central nervous system.

Figure 4 shows that the discrimination difference between the case of the increase and the decrease in the dip-frequency interval is observed over $600 \mathrm{~Hz}$, especially $1,000 \mathrm{~Hz}$, in the dip-frequency interval of Sound 1. This is because, as Bilsen and Ritsma point out, pitch sensation degrades rapidly for two sound intervals at shorter than 
$1.25 \mathrm{~ms}$, or longer than $20 \mathrm{~ms}$ [25]. The $20 \mathrm{~ms}$ in time delay means approximately $600 \mathrm{~Hz}$ in dip-frequency interval.

Figure 5 shows that the perceivable distance ratio to the dip-frequency interval is almost constant. This indicates that an instantaneous $\pm 3 \%$ change in the dip-frequency interval may give subjects a sensation of objects moving. However, the impression of tone coloration may not be the same between the blind and the sighted. Thus subjects other than the auditory-enhanced blind need to train to recognize obstacles by means of auditory stimuli.

\section{IMPRESSION ANALYSIS OF TIMBRE CHANGE ACCOMPANIED BY OBSTACLE DISTANCE CHANGE}

The preceding section shows that the blind and the sighted are the same in difference limen with regard to a change in the dip-frequency interval. However, the blind can practically recognize the direction of loudspeakers [35] and the existence of surrounding obstacles [36] more acutely than the sighted. This superiority results not from the peripheral sensation process, but from the central perception process of timbre change. This process of perception emerges as a psychological impression. Therefore, it is hypothesized that subjective impressions regarding timbre change among the blind differs from that among the sighted. The acoustic factors of the obstacle sense may be determined by a difference of impression between the two groups. In this section, we carry out an impression analysis among the blind and the sighted using the Semantic Differential Method (SD method).

\subsection{Subjects}

Six congenital visually impaired people aged 22-33 years old participated in the experiment. Five are congenitally blind, the other is acquired blind. Acquired blind subjects congenitally had visual disorders such as amblyopia and had been depended on as well as visual information before getting totally blind in midstream. Five are totally blind, the other can only recognize the existence of light stimuli. Four are males and two are females. As the sighted control group, two males and four females aged 23-42 years old participated. Sighted subjects forced to be blindfolded during this experiment. All of the subjects have normal hearing; within normal limits $(-20 \mathrm{~dB}$ HL) as defined by pure-tone audiometry. In addition, experimenter explains the contents of the experiment to subjects and all subjects agree to participation.

\subsection{Method}

As shown in the experimental setup in Fig. 6, the obstacle (a wooden board measuring $50 \times 70 \times 0.24 \mathrm{~cm}$ ) is placed $8.0 \mathrm{~m}$ away from the loudspeaker (TANOI system800) in a semianechoic chamber (size: $6.0 \times 6.0 \times$

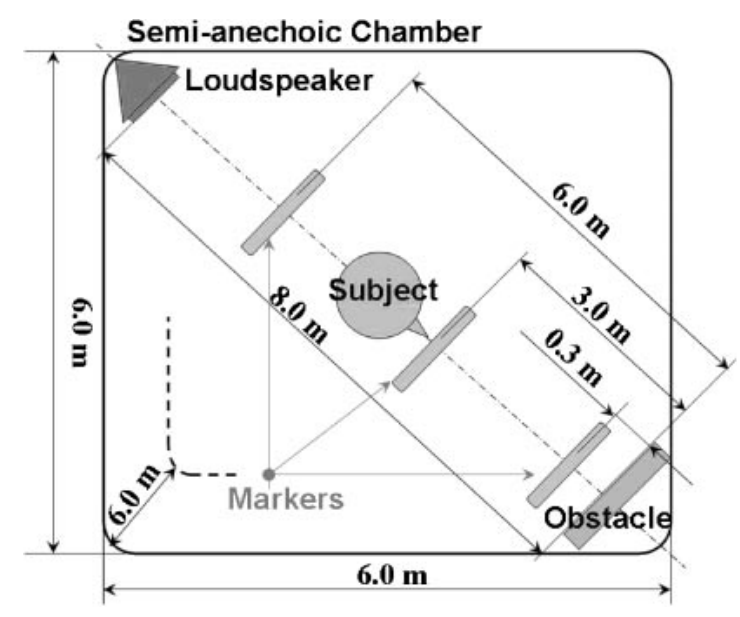

Fig. 6 Experimental setup of the impression analysis of timbre change.

$6.0 \mathrm{~m}$, background noise level: $30 \mathrm{~dB}$ ). Two markers are positioned at Points $\mathrm{A}$ and $\mathrm{B}$ ( $\mathrm{A}$ is nearer to the loudspeaker than $B$ ) between the loudspeaker and the obstacle to inform the subject of the starting and stopping positions. The combinations used for Points $A$ and $B$ are $6 m-3 m$, $3 \mathrm{~m}-0.3 \mathrm{~m}$ and $6 \mathrm{~m}-0.3 \mathrm{~m}$. According to the findings of a series of studies, approximately $3 \mathrm{~m}$ from the obstacle is defined as the position of "first perception," while approximately $0.3 \mathrm{~m}$ is defined as "final appraisal" [7,9]. This experiment is designed to investigate the impression change when a listener approaches these two positions.

The experimental procedure is as follows: First, a subject is placed at Point A, facing the obstacle. Second, white noise is played over the loudspeaker (volume: hearing level $+30 \mathrm{~dB}$ ). Third, the subject is asked to walk to Point B. The subject then evaluates the impression of timbre change by the adjective scales as shown in Table 4 on a scale of -3 to 3 ; scale 0 should not be rated as "little as possible." These adjective scale sets A-B mean - to + direction, e.g. "deep" corner means minus side, while "metallic" means plus side in the case of "deep-metallic." The authors picked these adjective scales in reference to Namba's study [37,38]. The order of combination of Points A and B is randomized for each subject. Subjects are allowed to walk back and forth toward the obstacle and to listen to the sound as they like: the average of taskelapsed time was approximately one minute. The experiment consists of 1 set for training and 2 sets in each condition (totally 6 sets) for the actual tests.

The evaluation results are processed by factor analysis based on the SDM. On the assumption of a dependence among factors for each other, either a maximum-likelihood method or a Promax rotation are applied for factorextraction and rotation, respectively. After finishing their tasks, the subjects are asked to describe their feelings as to the sound impression change and evaluation criteria. 


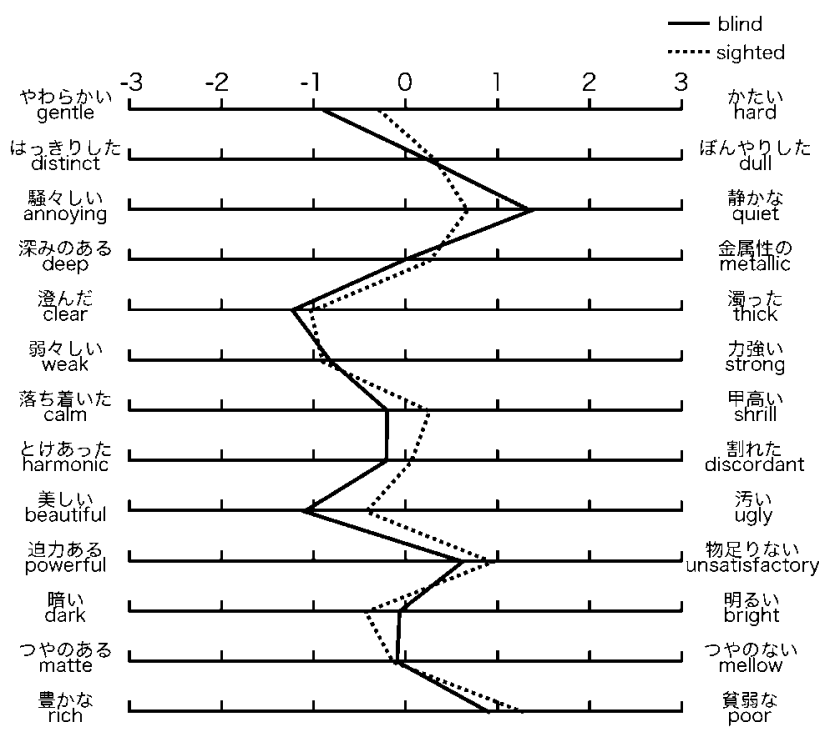

Fig. 7 Semantic profiles for blind and sighted groups.

Table 1 Eigenvalues of factor analysis.

\begin{tabular}{ccc}
\hline Factors & Eigenvalue (Sighted) & Eigenvalue (Blind) \\
\hline 1 & 4.652 & 6.455 \\
2 & 2.775 & 2.932 \\
3 & 1.811 & 1.106 \\
4 & 1.139 & 0.887 \\
5 & 0.777 & 0.513 \\
\hline
\end{tabular}

\subsection{Result}

The mean semantic profiles for blind and sighted groups in three conditions shown in Fig. 7 indicated that the evaluation tendency of the blind were similar to that of the sighted with the exception items such as "calm-shrill" and "harmonic-discordant."

The corresponding eigenvalues to the number of factors are shown in Table 1. The number of factors with an eigenvalue greater than 1 , are 4 in the sighted and 3 in the blind. However, because a great deal of researches for timbre impression discuss cases with 3 factors [38-40], similarly we also discuss cases of 3 factors.

The factor-pattern matrix and factor-correlation matrix of the sighted are shown in Tables 2 and 3, respectively. Cronbach's $\alpha$ for the scales in this group equals to 0.7052 .

Adjective scales that are highly related to Factor 1 are "weak-strong," "powerful-unsatisfactory," "annoyingquiet" and "harmonic-discordant." Thus, this factor can be interpreted as the factor of "powerful" factor that is quantitatively represented. Factor 2 shows high loadings for "matte-mellow," "clear-thick" and "beautiful-ugly." This factor can be called the factor of "cleanness." The third-factor-related adjective scales are: "calm-shrill," "dark-bright," "gentle-hard," "deep-metallic," and "distinct-dull." This factor can be termed the "metalic" factor.
Table 2 Factor-structure matrix (sighted group).

\begin{tabular}{crrr}
\hline \multirow{3}{*}{ Adjective scale } & \multicolumn{3}{c}{ Factor } \\
\cline { 2 - 4 } & \multicolumn{1}{c}{1} & \multicolumn{1}{c}{2} & \multicolumn{1}{c}{3} \\
\hline week-strong & $\underline{-0.916}$ & 0.017 & 0.243 \\
powerful-unsatisfactory & $\underline{0.856}$ & -0.293 & -0.066 \\
annoying-quiet & $\underline{0.822}$ & -0.068 & -0.238 \\
harmonic-discordant & $\underline{-0.522}$ & -0.138 & 0.365 \\
matte-mellow & 0.156 & $\underline{0.986}$ & -0.058 \\
clear-thick & -0.162 & $\underline{0.779}$ & 0.151 \\
beautiful-ugly & -0.221 & $\underline{0.777}$ & -0.169 \\
clam-shrill & 0.038 & 0.393 & $\underline{0.738}$ \\
dark-bright & 0.360 & 0.039 & -0.728 \\
gentle-hard & -0.440 & -0.111 & $\underline{0.663}$ \\
deep-metallic & -0.205 & -0.278 & $\underline{0.658}$ \\
distinct-dull & 0.475 & -0.203 & $\underline{-0.523}$ \\
rich-poor & 0.176 & 0.051 & -0.019 \\
\hline Proportion of & 38.053 & 23.216 & 13.406 \\
variance explained [\%] & & & \\
\hline
\end{tabular}

Table 3 Factor-correlation matrix (sighted group).

\begin{tabular}{crrr}
\hline Factor & 1 & 2 & \multicolumn{1}{c}{3} \\
\hline 1 & 1.000 & -0.023 & -0.094 \\
2 & -0.023 & 1.000 & 0.001 \\
3 & -0.094 & 0.001 & 1.000 \\
\hline
\end{tabular}

These tendencies are the same as in many studies such as Sone's and Namba's [38,39].

The factor-pattern matrix and factor-correlation matrix of the blind are shown in Tables 4 and 5, respectively. Cronbach's $\alpha$ for the scales in this group equals to 0.7075 .

Adjective scales that are highly related to Factor 1 are "deep-metallic," "calm-shrill," "dark-bright," "weakstrong," "powerful-unsatisfactory," "annoying-quiet" and "beautiful-ugly." This factor can be termed the factor of "sound timber" that can be only qualitatively represented. Furthermore, it includes the main related scales of all factors in the sighted group: "weak-strong," "powerful-satisfactory" and "annoying-quiet" corresponds to Factor 1 of the sighted, "beautiful-ugly" corresponds to Factor 2 of the sighted and "deep-metallic," "calm-shrill" and "dark-bright" corresponds to Factor 3 of the sighted. Factor 2 shows high loadings for "rich-poor," "mattemellow" and "harmonic-discordant." This factor can be interpreted as sound's "harmonic" factor because a sound's harmonics change in the case of the high sound's coherence. The third-factor-related adjective scales are: "gentle-hard" and "distinct-dull." This factor can be termed the "intelligibility" factor. The results indicate that the sighted tend to focus mainly on the quantitatively represented changes such as pitch and loudness of the sounds, while the blind are inclined to focus not only on the 
Table 4 Factor-pattern matrix (blind group).

\begin{tabular}{crrr}
\hline \multirow{2}{*}{ Adjective scale } & \multicolumn{3}{c}{ Factor } \\
\cline { 2 - 4 } & \multicolumn{1}{c}{1} & \multicolumn{1}{c}{2} & \multicolumn{1}{c}{3} \\
\hline deep-metallic & $\underline{0.846}$ & 0.059 & 0.137 \\
calm-shrill & $\underline{\underline{0.791}}$ & 0.027 & 0.213 \\
dark-bright & $\underline{\underline{0.785}}$ & -0.389 & 0.182 \\
weak-strong & $\underline{\underline{0.780}}$ & 0.027 & 0.532 \\
powerful-unsatisfactory & $\underline{-0.769}$ & -0.140 & -0.466 \\
annoying-quiet & $\underline{-0.760}$ & -0.352 & -0.331 \\
beautiful-ugly & $\underline{0.652}$ & 0.579 & -0.019 \\
rich-poor & -0.148 & $\underline{0.908}$ & -0.183 \\
matte-mellow & 0.065 & -0.897 & -0.096 \\
harmonic-discordant & 0.234 & $\underline{0.699}$ & 0.217 \\
gentle-hard & 0.345 & 0.256 & 0.872 \\
distinct-dull & -0.435 & -0.023 & -0.703 \\
clear-thick & -0.055 & -0.049 & 0.344 \\
\hline Proportion of & 49.656 & 22.552 & 8.506 \\
variance explained [\%] & & &
\end{tabular}

Table 5 Factor-correlation matrix (blind group).

\begin{tabular}{crrc}
\hline Factor & \multicolumn{1}{c}{1} & 2 & 3 \\
\hline 1 & 1.000 & -0.030 & 0.065 \\
2 & -0.030 & 1.000 & 0.044 \\
3 & 0.065 & 0.044 & 1.000 \\
\hline
\end{tabular}

quantitative sound change, but also on qualitative impressions in the sound changes.

\subsection{Discussion}

For both the sighted and the blind group, the extracted factors are the same and are different from the conventional studies such as $[38,41]$. The blind may be different in terms of what kind of sound characteristics they focused upon.

Factors 2 and 3 in the blind group are termed the factors of harmonics and intelligibility. In this experiment, white noise as a stimuli resulted in the generation of ripple noise due to the interference of direct sound and reflected sound from an obstacle. Increasing the dip-to-dip interval in the frequency response of ripple noise corresponding to the approaching obstacle raises the harmonic tonal pitch referred to as "repetition pitch" [19]. Thus, the reason for the extraction of a harmonics factor is that the blind focused on the repetition pitch of coloration, which is related to obstacle distance.

However, the extraction of the intelligibility factor may be caused by the less ambiguous presence of obstacle as the subject approaches the obstacle. Approximately $3 \mathrm{~m}$ from the obstacle is defined as the position of "first perception," while approximately $0.3 \mathrm{~m}$ is "final appraisal." The former and latter positions are the points for starting to perceive the existence of an obstacle and for predicting contact with the obstacle when approaching it, respectively $[7,9]$.
In addition, Kate applied the perception of coloration in filtered Gaussian noise to the central spectrum model [27]. Kate reported that maximum sensitivity occurs for delays at $2 \mathrm{~ms}$ : the obstacle-to-subject distance at about $0.3 \mathrm{~m}$ : the position of final appraisal. Bilsen reported an experiment using an analog noise generator with a tape-loop delay device to realize comb-filters and got a similar result [25]. They also indicated that pitch sensation degrades for two sound intervals longer than $20 \mathrm{~ms}$ : the position of first perception. In other words, one of the main reasons the blind can perceive it is dangerous to approach nearer to an obstacle is because coloration is at its highest sensitivity at final appraisal. Thus, the factor of intelligibility is related to the impression of obstacle unambiguity as determined by a change in timbre of coloration.

As a result, the following factors are obtained in the experiment; factors of "power," "cleanness" and "metallic" among the sighted, and factors of "sound timber," "harmonics" and "intelligibility" among the blind. Thus, the sighted tend to focus mainly on the quantitatively represented changes such as pitch and loudness of the sounds, while the blind are inclined to focus not only on the quantitative sound change, but also on qualitative impressions in the sound changes.

Abe et al. carried out a factor analysis for the perception of environmental sounds with adjective scales of feeling associated with sound and information of sound such as spatial characteristics, as well as adjectives describing sound quality [42]. They obtained the factors of not only cleanness, brightness (almost synonymous with metallic), and quantity (almost synonymous with potency), but also factors related to localization information, soundsource information, and the importance of existence and memory. Some investigators found a better ability among the blind for localization in the horizontal plain of signals and obstacles $[11,12,35]$. Our study did not employ these adjective scales for the spatial information of sounds, but factors of localization information and sound-source information could be highly related to the sound impression that concerns obstacle sense by the blind. Since it is assumed that the qualitative impressions are related to distances of the obstacles from the blind, we carry out a comparative experiment regarding the obstacle-distance perception.

\section{EXPERIMENT OF AUDITORY DISTANCE PERCEPTION}

The preceding section discusses matters what plays the main role in the process of obstacle sense: peripheral nerves, or the central nervous system. In this section, we tried to observe the reflection of the central process to the obstacle sense by carrying out an experiment regarding distance perception in the blind and the sighted. The 
purpose of this section is to ascertain that the blind can more exactly answer the obstacle-distance than the sighted because the blind have more cues for recognition of there surroundings than the sighted.

\subsection{Subject}

Four congenital visually impaired males aged 23-33 years old participated in the experiment. Three are totally blind, and one can only recognize the existence of light stimuli. One male and four females aged 18-23 years old participated as the sighted control group. All of the subjects have normal hearing; within normal limits ( $-20 \mathrm{~dB} \mathrm{HL})$ as defined by pure-tone audiometry. Before starting the experiment, all of the subjects put on an eye mask and wear an ear muff to shut out visual and auditory information. In addition, experimenter explains the contents of the experiment to subjects and all subjects agree to participation.

\subsection{Experimental Setup}

The experimental arrangement is shown in Fig. 6, apart from the fact that the markers are removed and a thick blanket is laid over the floor between the loudspeakers and the obstacle to prevent the subjects from hearing their own footsteps. The obstacle is a $50 \mathrm{~cm} \times 70 \mathrm{~cm} \times 24 \mathrm{~mm}$ (width $\times$ height $\times$ thickness) wooden board that is placed to the front of the subject at the same height as the center height of the subject's head and the obstacle. Subject stood at the center of the chamber or started to walk from the center in each trial. The distance of the obstacle from the subject was variable. Subjects could set the sound intensity of the loudspeaker at a comfortable level ( $45 \sim 60 \mathrm{~dB}$ SPL) at the beginning of the experiment.

\subsection{Method}

Before starting each trial, an experimenter moves the obstacle to default position; at a distance of $1 \mathrm{~m}, 2 \mathrm{~m}, 4 \mathrm{~m}$, or $6 \mathrm{~m}$ from the obstacle. First, a white noise (frequency range: $20 \mathrm{~Hz} \sim 20 \mathrm{kHz}$ ) is generated from the loudspeaker, and then the subject takes an ear muff off and try to walk to the half distance as far as the default position. For example, the subject tries to move to $2.0 \mathrm{~m}$ from the obstacle when an experimenter asks the subject to start moving, when the default position is $4.0 \mathrm{~m}$ from the obstacle. At that time, the subject are allowed to move back and forth while walking, but not allowed to produce sounds such as footsteps and vocal utterances. Then, the experimenter checks his arrival distance from the obstacle and requests the subject to wear an ear muff again. Before going on to the next trial, obstacle distance is changed by the experimenter.

The experiment was composed of 2 sets for training and 24 sets for actual test.

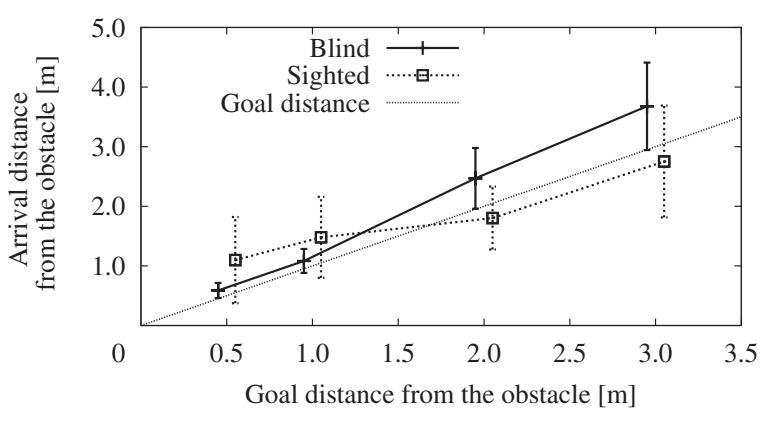

Fig. 8 Results of arrival distance from the obstacle. Marks for the blind and the sighted groups are plotted at $\pm 0.05 \mathrm{~m}$ points from the setup of the experimental configured distance transverse to each other to facilitate visualization. The dotted line represents the goal distance between the subject and the obstacle.

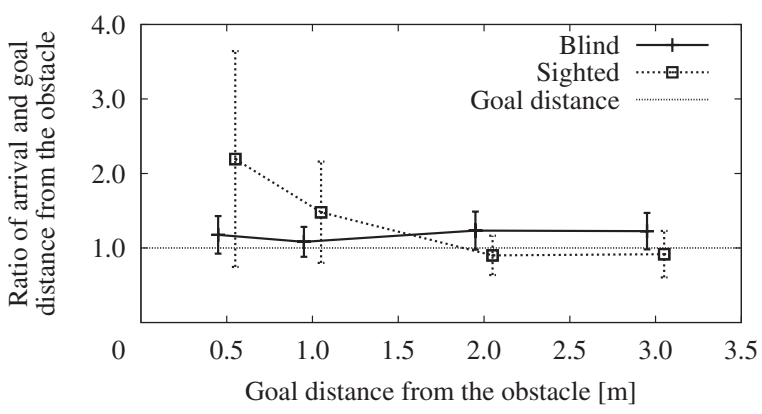

Fig. 9 Ratio of arrival and goal distance in relation to the goal distance from the obstacle. Marks for the blind and the sighted groups are plotted at $\pm 0.05 \mathrm{~m}$ points from the setup of the experimental configured distance transverse to each other to facilitate visualization. The dotted line represents the goal distance between the subject and the obstacle.

\subsection{Result and Discussion}

The arrival distances from the obstacle for the blind and the sighted groups are shown in Fig. 8. The sighted group localized a further distance than the goal distance when the goal distance was within and equal to $1.0 \mathrm{~m}$, while it achieved a nearer distance when the goal distance was more than and equal to $2.0 \mathrm{~m}$. The blind group localized a generally further distance than the goal distance. In addition, a remarkable variability in distance localization was observed in the sighted group, especially within and equals to $1.0 \mathrm{~m}$ of the goal di stance.

Figure 9 shows the ratio of arrival and goal distance in relation to initial distance from the obstacle. The blind group localized a position whose ratio from the goal distance remained constant.

The result of arrival time for a subject reaching the required position as illustrated by Fig. 10 shows that the blind took less time to localize than the sighted. 


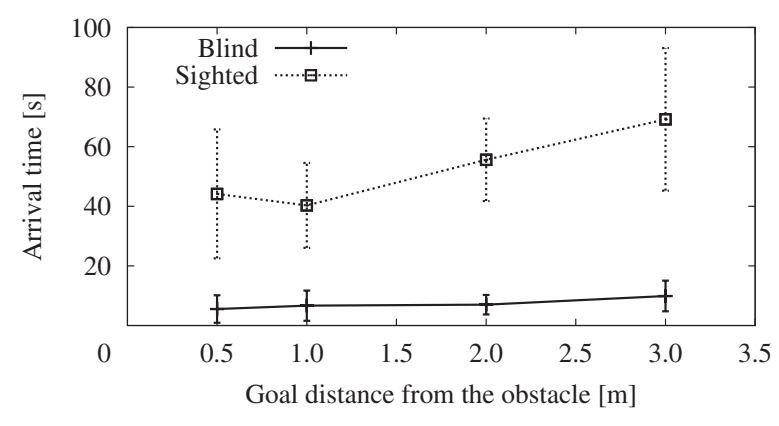

Fig. 10 Arrival time of subject's reaching the required position for the blind and the sighted groups.

Localized distance for the sighted group as shown in Figs. 8 and 9 are almost the same as that for the sighted group, as reported by Rosenblum [43]. The tendency for a decrease in the distance ratio as against an increase the goal distance among the sighted is due to the inconsistency of relying on which of two localization cues (acoustical or somatosensory) for the obstacle. However, the blind localized less variably in the distance ratio as shown in Fig. 9 because they consistently depended on one acoustical cue: coloration caused by ripple noise. They stopped a little bit further than the goal distance. This phenomenon might be caused by a habitually conscious act to avoid danger.

\section{GENERAL DISCUSSION}

The results of the first two experiments as mentioned at sections 3 and 4 show that there is no significant difference in the peripheral nervous system between the sighted and the blind, but that there are differences in the central process such as obstacle localization using acoustic cues. This central process results from the training of the spatial hearing because of the long-time complete deprivation of visual sense. Rauschecker found that binocularly deprived cats improve their auditory localization, and that their anterior ectosylvian visual areas are taken over by auditory inputs: auditory spatial tuning in the visual cortex is observed [44]. Lessard et al. reports that blind people with residual peripheral vision localize sounds less accurately than both the sighted and the total blind [45]. Thus, the development of auditory obstacle perception might be dependant on the complete deprivation of eyesight.

We now specifically discuss the role of the central process in the obstacle sense. It has been reported that higher activity in the striate and prestriate cortical areas is observed with the activation of the lateral cortex among the blind than among blindfolded sighted in auditory tasks [46-48]. Some investigators found that early blind people can localize azimuth with equal or higher accuracy, while they can localize elevation less accurate than the sighted [49,50]. Zwiers et al. successively stated that auditory localization for elevation needs optimal calibration to elevation-related spectral pinna cues, while azimuth localization can be calibrated without vision information [50]. Lewald et al. reported the posterior parietal cortex is specifically involved in azimuthal angles of sound and is a part of the "where" stream in the cortical processing of auditory information [51]. In our second experiment, an "intelligibility" factor was extracted from the blind group, which was caused by the following process after perceiving a timbre change. Thus, this factor is probably related to the visual and posterior parietal as well as auditory cortices.

The blind localize more constant in the distance ratio of obstacle and subject, and take a shorter time than the sighted, as shown in Figs. 9 and 10. This differences also probably results from more effective interactions among the auditory, visual and posterior parietal cortices. Hausfeld suggests that an improvement in echo perception by the sighted occurs after the first few trials in recognizing simple shapes and material difference of obstacles [52]. Thus, the generation of an efficient central interaction is developed by all in an effective condition for auditory localization training, such as complete deprivation of eyesight at most, or even under a similar condition when temporally without visual information. These training conditions are effectively realized by conventional acoustic virtual reality systems such as $[53,54]$. Thus, if acoustic virtual reality systems can present virtual acoustical obstacles to the blind, this system will also present the newly realistic sensation to the sighted. In addition, our result indicates that the sighted would potentially perceive obstacles aurally like the blind. If the sighted train the auditory obstacle perception over long period without visual information, they can probably obtain as useful obstacle sense as the blind.

Zwiers et al. found that the early blind localize elevation less accurately than the sighted [50], while there are a number of blind mobility aids that acoustically support obstacle detection in the horizontal plane $([2,3,5]$, et al.). Support for obstacle detection in the frontal plane relies on tactile devices such as white canes or canes equipped with sensors [55], and these mainly help the blind to perceive obstacle on the ground. However, these devices face a difficulty when trying to detect obstacles not directly on the ground at a height somewhere between knee- and chest-height. Thus, future blind mobility aids can contribute to improving the quality of life by enabling the blind to perceive obstacles even in the frontal plain with utilizing the obstacle sense positively as well as without preventing auditory obstacle perception. When this concept reflects to blind mobility aid, blinds who are either well or not well in obstacle sense can use it effectively. Although the way to assist the obstacle sense actively remains unknown only in our study, we will propose the better model of the blind mobility aid based on our findings in addition to the other studies especially Zwiers' results. 


\section{CONCLUSION}

Three experiments related to obstacle sense by the blind were carried out in order to clarify its perceptual process. The result for the discrimination ability in various coloration changes implied the same discrimination ability between blind and sighted groups. Thus, there was little difference in the peripheral auditory system between them, such as perception differences of various dip-frequency intervals in coloration.

In the second experiment of sound impression change while approaching to an object on a flat surface, the extracted factors were different between the blind and the sighted. This result suggested that the blind could detect the obstacle by perceiving the quality changes of sound impressions. It means that the obstacle sense would be performed in the central auditory system.

In the third experiment, the experiment of auditory distance perception of obstacle is carried out for observing the reflection of the central process to spacial perception. The results indicated the blinds' superiority in central process: more accurate in the localized distance and shorter-time in the distance localization than the sighted. These results implied that the training of obstacle sense would be possible for the untrained acquired or justacqiured blind as well as the sighted. In addition, we discussed that the central process among the blind resulted from more effective interactions among the auditory, visual and posterior parietal cortices.

Finally, from the results of the three experiments and past studies, we discussed new concepts for blind mobility aids as well as the obstacle sense training method.

\section{ACKNOWLEDGEMENT}

This work is supported by JSPS Grant-in-Aid for Scientific Research (S), 17100009. We are grateful to Professor Shigeki Sagayama for his encouragement.

\section{REFERENCES}

[1] Y. Seki, "Acoustical design of city for the visually handicapped," J. Acoust. Soc. Jpn. (J), 54, 387-392 (1998).

[2] T. Ifukube, T. Sasaki and C. Peng, "A blind mobility aid modeled after echolocation of bats," IEEE Trans. Biomed. Eng., 38, 461-465 (1991).

[3] N. Pressey, "Mowat sensor," Focus, 3, 35-39 (1977).

[4] L. Kay, "The design and evaluation of a sensory aid to enhance spatial perception of the blind," Dept. Elect. Eng., Univ. Canterbury, New Zealand, Rep. 21 (1973).

[5] L. Kay, "Auditory perception of objects by blind persons, using a bioacoustic high resolution air sonar," J. Acoust. Soc. Am., 107, 3266-3275 (2000).

[6] S. Cardin, D. Thalmann and F. Vexo, "A wearable system for mobility improvement of visually impaired people," Vis. Comput., 23, 109-118 (2007).

[7] M. Supa, M. Cotzin and K. M. Dallenbach, " "Facial vision": The perception of obstacles by the blind," Am. J. Psychol., 57,
133-183 (1944).

[8] P. Worchel and K. M. Dallenbach, " "Facial vision": Perception of obstacles by the deaf-blind," Proc. Int. Congr. Technol. Blindness, 60, 502-553 (1947).

[9] M. Cotzin and K. M. Dallenbach, "Facial vision": The role of pitch and loudness in the perception of obstacles by the blind," Am. J. Psychol., 63, 485-515 (1950).

[10] Y. Seki, T. Ifukube and Y. Tanaka, "Relation between the reflected sound localization and the obstacle sense of the blind," J. Acoust. Soc. Jpn. (J), 50, 289-295 (1994).

[11] W. N. Kellogg, "Sonar system of the blind," Science, 137, 399-404 (1962).

[12] C. E. Rice, "Human echo perception," Science, 155, 656-664 (1967).

[13] C. E. Rice and S. H. Feinstein, "Sonar system of the blind: Size discrimination," Science, 148, 1107-1108 (1965).

[14] T. Stroffregen and J. Pittenger, "Human echolocation as a basic form of perception and action," Ecol. Psychol., 7, 181216 (1995).

[15] D. Diderot, "Letter on the blind," (1749), in Thoughts on the Interpretation of Nature and Other Philosophical Works, D. Diderot and D. J. Adams, Eds. (Clinamen Pr Ltd., Manchester, 2000).

[16] S. P. Hayes, Contributions to a Psychology of Blindness (American Foundation for the Blind, New York, 1941).

[17] Y. Seki, T. Ifukube and Y. Tanaka, "The influence of sound insulation effect on obstacle sense of the blind," J. Acoust. Soc. Jpn. (J), 50, 382-385 (1994).

[18] I. G. Bassett and E. J. Eastmond, "Echolocation: Measurement of pitch versus distance for sounds reflected from a flat surface," J. Acoust. Soc. Am., 36, 911-916 (1964).

[19] W. Yost and R. Hill, "Models of the pitch and pitch strength of ripple noise," J. Acoust. Soc. Am., 66, 400-410 (1979).

[20] W. A. Yost, "Pitch and pitch discrimination of broadband signals with rippled power spectra," J. Acoust. Soc. Am., 63, 1166-1175 (1978).

[21] F. L. Wightman and D. M. Green, "The perception of pitch," Am. Sci., 62, 208-215 (1974).

[22] R. D. Patterson, "The effects of relative phase and the number of components on residue pitch," J. Acoust. Soc. Am., 53, 1565-1572 (1973).

[23] R. D. Patterson and F. L. Wightman, "Residue pitch as a function of component spacing," J. Acoust. Soc. Am., 59, 1450-1459 (1976).

[24] W. A. Yost, R. Patterson and S. Sheft, "A time domain description for the pitch strength of iterated rippled noise," $J$. Acoust. Soc. Am., 99, 1066-1078 (1996).

[25] F. A. Bilsen and R. J. Ritsma, "Some parameters influencing the perceptibility of pitch," J. Acoust. Soc. Am., 47, 469-475 (1970).

[26] Y. Ando and H. Alrutz, "Perception of coloration in sound fields in relation to the autocorrelation function," J. Acoust. Soc. Am., 71, 616-618 (1982).

[27] J. M. Kates, "A central spectrum model for the perception of coloration in filtered gaussian noise," J. Acoust. Soc. Am., 77, 1529-1534 (1985).

[28] K. Krumbholz, K. Maresh, J. Tomlinson, R. D. Patterson, A. Seither-Preisler and B. Lutkenhoner, "Mechanisms determining the salience of coloration in echoed sound: Influence of interaural time and level differences," J. Acoust. Soc. Am., 115, 1696-1704 (2004).

[29] Y. Seki and K. Ito, "Coloration perception depending on sound direction," IEEE Trans. Speech Audio Process., 11, 817-825 (2003).

[30] J. Terhune, "Sound localization abilities of untrained humans 
using complex and sinusoidal sounds," Scand. Audiol., 3, 115120 (1974).

[31] M. Bross and M. Borenstein, "Temporal auditory acuity in blind and sighted subjects: A signal detection analysis," Percept. Mot. Skills, 55, 963-966 (1982).

[32] I. Starlinger and W. Niemeyer, "Do the blind hear better? Investigations on auditory processing in congenital or early acquired blindness. I. Peripheral functions," Audiology, 20, 503-509 (1981).

[33] W. Niemeyer and I. Starlinger, "Do the blind hear better? Investigations on auditory processing in congenital or early acquired blindness. II. Central functions," Audiology, 20, 510 515 (1981).

[34] L. Benedetti and M. Loeb, "A comparison of auditory monitoring performance in blind subjects with that of sighted subjects in light and dark," Percept. Psychophys., 11, 10-16 (1972).

[35] C. Muchnik, M. Efrati, E. Nemeth, M. Malin and M. Hildesheimer, "Central auditory skills in blind and sighted subjects," Scand. Audiol., 20, 19-23 (1991).

[36] H. Lai and Y. Chen, "A study on the blind's sensory ability," Int. J. Ind. Ergon., 36, 565-570 (2006).

[37] S. Namba and S. Kuwano, Method of Psychological Measurement for Hearing Research (Corona Publishing Co. Ltd., Tokyo, 1998).

[38] S. Namba, S. Kuwano, K. Kinoshita and K. Kurakata, "Loudness and timbre of broad-band noise mixed with frequency-modulated sounds," J. Acoust. Soc. Jpn. (E), 13, 49-58 (1992).

[39] T. Sone, K. Kido and T. Nimura, "Lv80 analysis of descriptive adjectives for the evaluation of sounds," J. Acoust. Soc. Jpn. (E), 18, 320-326 (1962).

[40] R. Nishimura, T. Suenaga, Y. Suzuki and A. Tanaka, "Effects of sound detarioration on sound impression space," J. Acoust. Soc. Jpn. (J), 62, 63-72 (2008).

[41] M. Kuriyagawa, H. Yahiro and S. Kashiwagi, "Seven attributes in tone quality evaluation," J. Acoust. Soc. Jpn. (J), 34, 493-500 (1978).

[42] K. Abe, K. Ozawa, Y. Suzuki and T. Sone, "Evaluation of environmental sounds using adjectives describing sound quality, emotional state, and information carried by sounds," J. Acoust. Soc. Jpn. (J), 54, 343-350 (1998).

[43] L. D. Rosenblum, M. S. Gordon and L. Jarquin, "Echolocating distance by moving and stationary listeners," Ecol. Psychol., 12, 181-206 (2000).

[44] J. P. Rauschecker, "Compensatory plasticity and sensory substitution in the cerebral cortex," Trends Neurosci., 18, 36-43 (1995).

[45] N. Lessard, M. Pare, F. Lepore and M. Lassonde, "Early-blind human subjects localize sound sources better than sighted subjects," Nature, 395, 278-280 (1998).

[46] M. C. Wanet-Defalque, C. Veraart, A. De Volder, R. Metz, C. Michel, G. Dooms and A. Goffinet, "High metabolic activity in the visual cortex of early blind human subjects," Brain Res., 446, 369-373 (1988)

[47] P. Arno, A. G. De Volder, A. Vanlierde, M. C. WanetDefalque, E. Streel, A. Robert, S. Sanabria-Bohorquez and C. Veraart, "Occipital activation by pattern recognition in the early blind using auditory substitution for vision," Neuroimage, 13, 632-645 (2001).

[48] T. Kujala, M. Huotilainen, J. Sinkkonen, A. I. Ahonen, K. Alho, M. S. Hämäläinen, R. J. Ilmoniemi, M. Kajola, J. E. Knuutila, J. Lavikainen, O. Salonen, J. Simola, C.-G. Standertskjöld-Nordenstam, H. Tiitinen, S. O. Tissari and R. Näätänen, "Visual cortex activation in blind humans during sound discrimination," Neurosci. Lett., 183, 143-146 (1995).

[49] A. Dufour, O. Despres and V. Candas, "Enhanced sensitivity to echo cues in blind subjects," Exp. Brain Res., 165, 515-519 (2005).

[50] M. P. Zwiers, A. J. V. Opstal and J. R. M. Cruysberg, "A spatial hearing deficit in early-blind humans," J. Neurosci., 21, $1-5$ (2001).

[51] J. Lewald, H. Foltys and R. Töpper, "Role of the posterior parietal cortex in spatial hearing," J. Neurosci., 22, 1-5 (2002).

[52] S. Hausfeld, R. P. Power, A. Gorta and P. Harris, "Echo perception of shape and texture by sighted subjects," Percept. Mot. Skills, 55, 623-632 (1982).

[53] M. Kobayashi and M. Ohta, "Walking guide system for the visually impaired by using omni-vision sensor and threedimensional sound," Soc. Biomech. Jpn., 24, 123-125 (2000).

[54] Y. Seki, "Auditory obstacle perception and training," $J$. Acoust. Soc. Jpn. (J), 60, 325-329 (2004).

[55] S. Shoval, I. Ulrich and J. Borenstein, "Navbelt and the guidecane," IEEE Robot. Autom. Mag., 10, 9-20 (2003).

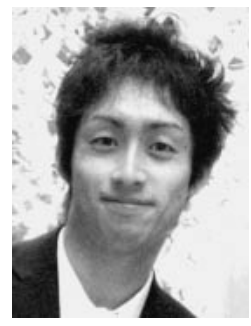

Takahiro Miura He received his Bachelors degree in mechanical engineering from Tohoku University, Sendai, Japan in 2005. Later, in March 2007, he received his Masters degree in information physics and computing from the University of Tokyo, Tokyo, Japan, where he is currently a doctoral course student. His research interests include assistive devices for the blind.

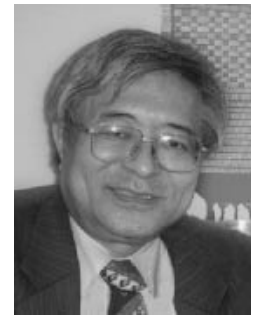

Teruo Muraoka He completed graduate studies in 1967. In the same year he joined Victor Company of Japan, Ltd. (JVC). He received his $\mathrm{PhD}$ from Kyushu University in 1978 after publishing a thesis on the carrier quadraphonic disc records CD-4. In 2001, he retired from JVC and assumed a professorship at Musashi Institute of Technology where he engaged in research on GHA, Multichannel sound field recording \& reproduction, analysis of stereophonic recording techniques etc, until retiring in 2006. He currently continues his research into the same themes at the Research Center of Advanced Science and Technology at the University of Tokyo.

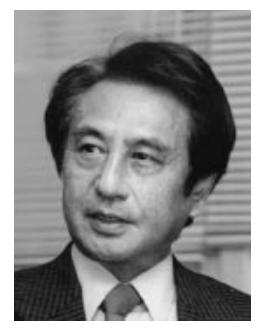

Tohru Ifukube $\mathrm{He}$ received the MS and Dr. Eng degrees in electronics from Hokkaido University in 1971 and 1977, respectively. He joined Research Institute of Applied Electricity, Hokkaido University, in 1971. He was a visiting Associate Professor at Stanford University, CA in 1984 and also he was a professor at Hokkaido University from 1989 to 2002 . He has been a professor at the Research Center for Advanced Science and Technology, University of Tokyo since 2002. He is a fellow of IEICE and a professor emeritus of Hokkaido University. His research interests include the analysis of the hearing, speaking and visual functions, and the design of aid devices for the disabled. Some of the aid devices have been used for the blind, the deaf and the speech disordered, and also have been applied to virtual reality and robotic systems. He published some books such as "Design of Voice Typewriter" (1983), "Sound-based Assistive Technology" (1997), "Evaluation of Virtual Reality" (Editor, 2000), and "Challenges of Assistive Technology" (2004). 